\title{
Magnetism and Interaction-Induced Gap Opening in Graphene with Vacancies or Hydrogen Adatoms: Quantum Monte Carlo Study
}

\author{
M. V. Ulybyshev ${ }^{1,2,3, *}$ and M. I. Katsnelson ${ }^{4,5, \dagger}$ \\ ${ }^{1}$ Institute of Theoretical Physics, University of Regensburg, Universitatsstrasse 31, D-93053 Regensburg, Germany \\ ${ }^{2}$ Institute for Theoretical Problems of Microphysics, Moscow State University, Moscow 119899, Russia \\ ${ }^{3}$ ITEP, B. Cheremushkinskaya street 25, Moscow 117218, Russia \\ ${ }^{4}$ Radboud University, Institute for Molecules and Materials, Heyndaalseweg 135, NL-6525AJ Nijmegen, The Netherlands \\ ${ }^{5}$ Theoretical Physics and Applied Mathematics Department, Ural Federal University, Mira str. 19, Ekaterinburg 620002, Russia
}

(Received 23 February 2015; published 15 June 2015)

\begin{abstract}
We study the electronic properties of graphene with a finite concentration of vacancies or other resonant scatterers by a straightforward lattice quantum Monte Carlo calculation. Taking into account a realistic long-range Coulomb interaction, we calculate the distribution of the spin density associated with midgap states and demonstrate antiferromagnetic ordering. An energy gap is open due to interaction effects, both in the bare graphene spectrum and in the vacancy or impurity bands. In the case of a 5\% concentration of resonant scatterers the latter gap is estimated to be $0.7 \mathrm{eV}$ and $1.1 \mathrm{eV}$ for graphene on boron nitride and freely suspended graphene, respectively.
\end{abstract}

DOI: 10.1103/PhysRevLett.114.246801

PACS numbers: 73.22.Pr, 05.10.Ln, 71.30.th

Defects enormously effect the electronic properties of graphene and other Dirac materials. In particular, vacancies in graphene are known to create midgap states $[1,2]$ that, together with the electron-electron interaction, can result in the appearance of magnetic moments and rich many-body phenomena (see the review of early work in Refs. [2,3] and recent experimental and theoretical papers [4-7]). Note that hydrogen adatoms and some univalent organic admolecules (resonant scattering centers) produce a very similar electronic structure [8]. Qualitatively, this is explained by the fact that the $s p^{3}$ state of the carbon atom originates from its bond with a univalent adatom or admolecule, making it unavailable for $p_{z}$ electrons ( $\pi$ orbitals) at energies at the neutrality point plus or minus several $\mathrm{eV}$; for these electrons such an atom is just cut from the lattice. Thus, in discussing "vacancies" we will also keep in mind these cases; moreover, they are even closer to the simple model of a vacancy as just a missed site in the honeycomb lattice (the model that will be used in our calculations) since the real vacancy produces very strong lattice distortions essentially effecting the electronic structure [9]. The case of a finite concentration of vacancies is quite complicated even at the single-particle level [10-12]. Here, we consider this case by taking into account a realistic model of the Coulomb interaction in graphene [13] via straightforward lattice quantum Monte Carlo (QMC) simulations. Keeping in mind what was said above, the best and easiest experimental realization would be partially hydrogenated graphene. We will study the antiferromagnetic phase transition driven by the presence of adatoms. It manifests itself in emergent magnetic moments concentrated near the adatoms, as well as in band gap opening. Both features of this phase transition are very important. The band gap, which is controllable via hydrogenation, offers an interesting possibility for prospective graphene applications in semiconductor devices. Emergent magnetic moments could be one of the reasons for the short spin relaxation time in graphene, which is an essential obstacle for producing efficient spintronic devices $[14,15]$. We emphasize that unlike the previous density functional theory study of vacancies in graphene $[9,16]$, here we treat a very large sample with a random (nonregular) distribution of vacancies using unbiased QMC calculations. Thus, we could estimate how close the vacancies influence each other in various geometrical configurations (for example, by a calculation of the variations of the magnetic moment). Also, we could extract the energy of the midgap state associated with any particular scatterer and observe the variations of these energies from one scatterer to another depending on its surroundings. These measurements give us an opportunity to estimate a realistic width for the midgap energy band for a fixed distribution of scatterers. The demonstrated possibility of QMC simulations of large samples with arbitrary positions of scatterers is even more important because in real samples adatoms can migrate and form clusters. This phenomenon is now the focus of intensive research $[17,18]$. Our technique can be applied to these real spatial configurations of scatterers. In principle, one can calculate the potential of interaction between adatoms by QMC calculations and model the formation of clusters, which is an interesting project for the future. Here, we restrict ourselves only to the case of a random distribution of defects.

We start with the tight-binding Hamiltonian for noninteracting electrons with a staggered mass term, which is essential in our simulations for the following reasons: (1) it eliminates the zero mode in the spectrum of quasiparticles, 
thus making the fermionic operator $M$ (see below) invertible and (2) it serves as a seed for the antiferromagnetic phase transition that we will study. The initial Hamiltonian without interaction and adatoms reads

$$
\begin{aligned}
\hat{H}_{t b}= & -t \sum_{\langle x, y\rangle}\left(\hat{a}_{y, \uparrow}^{\dagger} \hat{a}_{x, \uparrow}+\hat{a}_{y, \downarrow}^{\dagger} \hat{a}_{x, \downarrow}+\text { H.c. }\right) \\
& \pm \sum_{x} m\left(\hat{a}_{x, \uparrow}^{\dagger} \hat{a}_{x, \uparrow}-\hat{a}_{x, \downarrow}^{\dagger} \hat{a}_{x, \downarrow}\right)
\end{aligned}
$$

where $t=2.7 \mathrm{eV}$, the sum $\sum_{\langle x, y\rangle}$ goes over all pairs of nearest-neighbor sites of the graphene honeycomb lattice (we impose periodic spatial boundary conditions as in Refs. $[19,20])$, and the mass term has a different sign for different sublattices. Here, $\hat{a}_{x, \uparrow}^{\dagger}, \hat{a}_{x, \uparrow}$ and $\hat{a}_{x, \downarrow}^{\dagger}, \hat{a}_{x, \downarrow}$ are the creation or annihilation operators for spin up and spin down electrons at $\pi$ orbitals.

Next, we introduce the electrostatic interaction with potentials $\quad V_{x y}: \quad \hat{H}_{C}=\frac{1}{2} \sum_{x, y} V_{x y} \hat{q}_{x} \hat{q}_{y}, \quad$ where $\hat{q}_{x}=$ $\hat{a}_{x, \uparrow}^{\dagger} \hat{a}_{x, \uparrow}+\hat{a}_{x, \downarrow}^{\dagger} \hat{a}_{x, \downarrow}-1$ is the operator of an electric charge at lattice site $x$. The whole matrix $V_{x y}$ is constructed in the following way: at small distances [on site interaction $\left(V_{x x} \equiv V_{00}\right)$ and interactions with the nearest $\left(V_{01}\right)$, next-to-nearest $\left(V_{02}\right)$, and next-to-next-to-nearest $\left(V_{03}\right)$ neighbors] we use the potentials calculated by the constrained RPA method [13]; at larger distances we use the ordinary Coulomb $V_{x y}=V_{C} r_{01} / r_{x y}$. The parameter $V_{C}$ defines the strength of the Coulomb tail. Since $r_{01}$ is the distance between the nearest neighbors, $V_{C}$ is equal to the Coulomb repulsion energy at the distance of the conjugated $\mathrm{C}-\mathrm{C}$ bond. There is a small difference in the calibration in our model $(t=2.7 \mathrm{eV})$ and the calculations in Ref. [13], where the hopping parameter was $2.8 \mathrm{eV}$, but any possible changes in observable quantities due to this difference are definitely outside the accuracy of the applied QMC technique.

We use two sets of the interaction potentials. The first is called "ordinary potentials" and corresponds to freely suspended graphene (the dielectric constant of the environment is equal to 1). At small distances it is simply the set of potentials from Ref. [13]: $V_{00}=9.3 \mathrm{eV}, V_{01}=5.5 \mathrm{eV}$, $V_{02}=4.1 \mathrm{eV}$, and $V_{03}=3.6 \mathrm{eV}$. The strength of the Coulomb tail is defined by the ratio $V_{03}=V_{C} r_{01} / r_{03}$; thus, $V_{C}=7.2 \mathrm{eV}$. It is a simple continuous extension of the potentials at small distances. The second set is specified as "screened potentials" and corresponds to graphene at a substrate with a dielectric constant $\epsilon=4.5$, which is roughly the value reasonable for both boron nitride and $\mathrm{SiO}_{2}$. In this case the Coulomb tail is $(1+\epsilon) / 2=2.75$ times weaker while $V_{02}$ and $V_{03}$ are 1.5 times smaller. $V_{00}$ and $V_{01}$ are untouched since screening by the substrate should be irrelevant at a few interatomic distances.

As was already mentioned, the vacancy or adatom is modeled by setting to zero all hoppings to the vacant site.
We also exclude the vacant sites from the interaction term $H_{C}$ because they have constant zero charge. We employ a hybrid Monte Carlo algorithm, broadly used in lattice QCD. It was applied earlier for studies of graphene in Refs. [21,22], where so-called staggered fermions were used to model the low-energy effective field theory of graphene. This algorithm was developed further in Refs. [19,20,23]. First, we perform a Suzuki-Trotter decomposition of the partition function $\exp (-\beta H)$ in order to represent it as a functional integral over trajectories in Euclidean time. In order to get rid of the four fermionic terms in the Hamiltonian, we use a Hubbard-Stratonovich transformation and obtain the following partition function after integrating out fermionic fields:

$$
\operatorname{Tr} e^{-\beta \hat{H}} \cong \int \mathcal{D} \varphi_{x, n} e^{-S\left[\varphi_{x, n}\right]}\left|\operatorname{det}\left(M\left[\varphi_{x, n}\right]\right)\right|^{2},
$$

where $\varphi_{x, n}$ is the Hubbard-Stratonovich field for time slice $n$ and spatial coordinate $x . \delta \tau N_{t}=\beta$, where $\delta \tau$ is the step in Euclidean time, $N_{t}$ is the number of steps, $\beta$ is the inverse temperature, and $M$ is the fermionic operator (inverse fermionic Green's function at a given configuration of auxiliary field). We use its particular form [20]. It was discussed in Ref. [24] in more detail (including issues with the continuous limit $\delta \tau \rightarrow 0$ ). The particle-hole symmetry for graphene at the neutrality point makes the integration weight in Eq. (2) positive due to the appearance of the squared modulus in the determinant; thus, we have no fermionic sign problem [25]. For both sets of interelectron interaction potentials, the action of the HubbardStratonovich field $S\left[\varphi_{x, n}\right]$ is also of a positive definite quadratic form. Thus, we generate the configurations of $\varphi_{x, n}$ by a Monte Carlo method and calculate physical quantities as averages over the generated configurations. Here, we follow Refs. $[19,20,23]$ and use the so-called $\Phi$ algorithm.

We used lattices with spatial sizes $18 \times 18,24 \times 24$ and $36 \times 36$ in order to study finite-size effects. We studied a lattice with 5\% adatoms (in most of the calculations), scattered uniformly through the whole sample. Three different temperatures were studied: $T=0.5 \mathrm{eV}$ (corresponding to $\left.N_{t}=20\right), T=0.125 \mathrm{eV}\left(N_{t}=80\right)$, and $T=$ $0.0625 \mathrm{eV}\left(N_{t}=160\right)$. For all temperatures we generated configurations with four masses; for example, in the case of $T=0.125 \mathrm{eV}$ we used $m=0.05,0.1,0.15$, and $0.2 \mathrm{eV}$. Physical results are obtained via extrapolation to zero mass. In all calculations except for the energies of the midgap states we use "ordinary potentials."

According to the Lieb theorem for the Hubbard model $[2,26]$ the ground state for the case of vacancies equally distributed between two sublattices should be a spin singlet, and there are no physical reasons to expect that the longrange character of the Coulomb interactions can change this conclusion. Keeping in mind that a single vacancy or 
adatom induces a magnetic moment, one should consider the opportunity for antiferromagnetic ordering at a finite concentration (ferromagnetism is impossible). In this case the order parameter is the difference in average spin between sublattices (denoted as $A$ and $B$ in the formula): $\quad\langle\Delta n\rangle=\left\langle\left(1 / N_{A}\right) \sum_{x \in A}\left(\hat{a}_{x, \uparrow}^{\dagger} \hat{a}_{x, \uparrow}-\hat{a}_{x, \downarrow}^{\dagger} \hat{a}_{x, \downarrow}\right)-\right.$ $\left.\left(1 / N_{B}\right) \sum_{x \in B}\left(\hat{a}_{x, \uparrow}^{\dagger} \hat{a}_{x, \uparrow}-\hat{a}_{x, \downarrow}^{\dagger} \hat{a}_{x, \downarrow}\right)\right\rangle ; N_{A}$ and $N_{B}$ are the overall number of sites in the $A$ and $B$ sublattice, respectively. The results are presented in Fig. 1(a). In the case of the highest temperature $(0.5 \mathrm{eV})$ the order parameter is equal to zero in the physical limit of zero bare mass, disregarding the presence of adatoms. Only at the lower temperature $(0.125 \mathrm{eV})$ does the order parameter acquire a nonzero value in the presence of adatoms and it remains almost stable with a further decrease in temperature $(0.0625 \mathrm{eV})$. Figure 1(b) presents the temperature dependence of the antiferromagnetic order parameter. This calculation was also performed using one particular random distribution of adatoms for each concentration. One can clearly see a sharp transition at a certain critical temperature (Neel temperature). The results were fitted with a "step
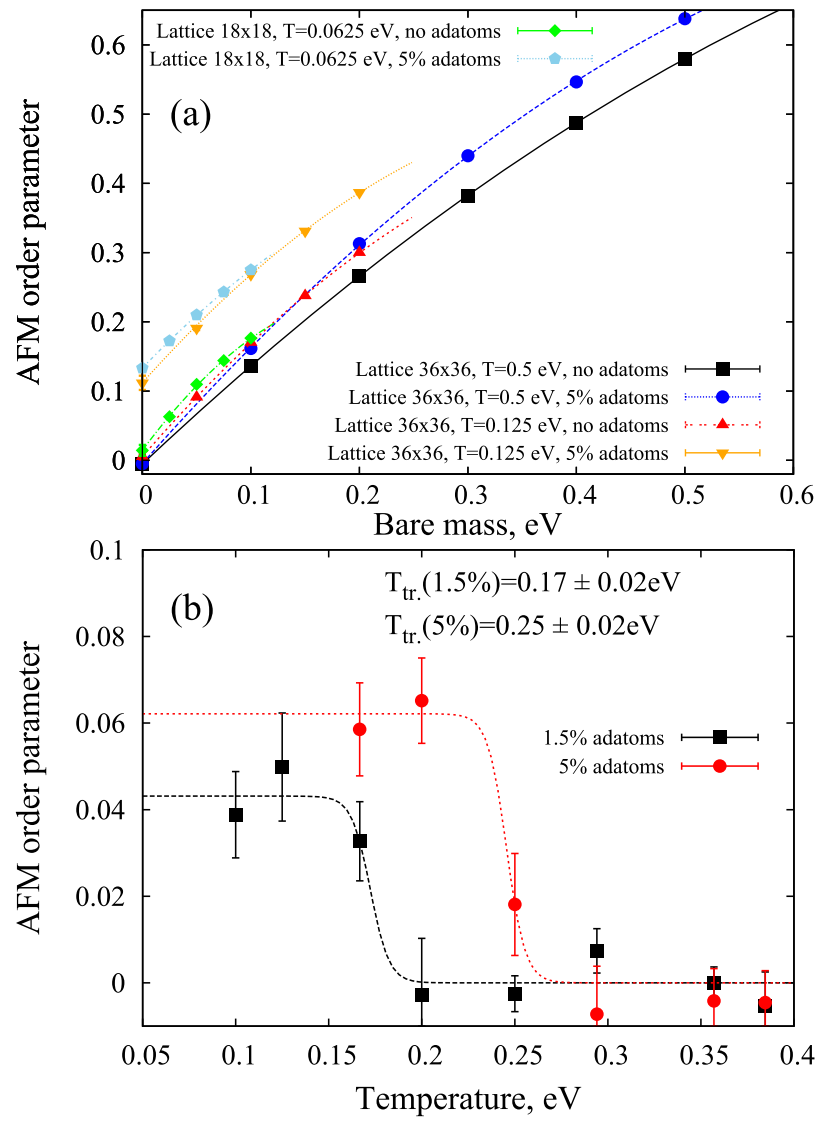

FIG. 1 (color online). (a) Antiferromagnetic order parameter at various temperatures, calculated on different lattices in the presence of adatoms and without them. (b) Temperature dependence of the antiferromagnetic order parameter in the zero bare mass limit. The calculation was performed on a $12 \times 12$ lattice. function" $\quad f(T)=C\{1-\tanh [b(T-a)]\}$, where the parameter $a$ gives us the value of the critical temperature. Thus, we have an estimation of the effective antiferromagnetic coupling (for a $1 \%$ concentration of defects), $J \sim 0.1 \mathrm{eV}$. This value is 2 orders of magnitude larger than the one estimated from recent experimental data for vacancies in graphene [27]. This is an important point, showing that the exchange interaction is probably very sensitive to the real electronic structure (we mentioned in the introduction that for real vacancies it is very strongly effected by atomic reconstruction) and that the use of the simplest one-band tight-binding model instead of fullelectron calculations can be dangerous in problems related to magnetism. Recent density functional calculations of exchange interactions in single-site hydrogenated or fluorinated graphene [28] predict complicated noncollinear magnetic ground states, in sharp contrast with the predictions of the Lieb theorem for the single-band model (saturated ferromagnetism). This issue requires further investigation.

The spatial distribution of the electron spin density is presented in Fig. 2. It represents the quantity $f_{x}=$ $\left\langle\hat{a}_{x, \uparrow}^{\dagger} \hat{a}_{x, \uparrow}\right\rangle$ at each lattice site. Since the particle-hole symmetry is unbroken, the equality $\left\langle\hat{a}_{x, \uparrow}^{\dagger} \hat{a}_{x, \uparrow}\right\rangle+$ $\left\langle\hat{a}_{x, \downarrow}^{\dagger} \hat{a}_{x, \downarrow}\right\rangle=1$ is satisfied exactly for each lattice site. This means that regions with positive $f_{x}$ have noncompensated spin up; negative $f_{x}$ corresponds to noncompensated spin down. It is clearly seen that antiferromagnetic order is generated in the vicinity of the adatoms. Moreover, one isolated adatom has a nonzero average spin (see the first row in Table I). These spins tend to be parallel for adatoms at one sublattice and antiparallel for adatoms at a different sublattice. If the adatoms are placed equivalently on both sublattices, they generate the same spin excess at both sublattices and thus the full spin will be close to zero. This means that, indeed, the statement of the Lieb theorem [26] remains correct in the case of long-range Coulomb interaction. A more detailed description of spin-spin

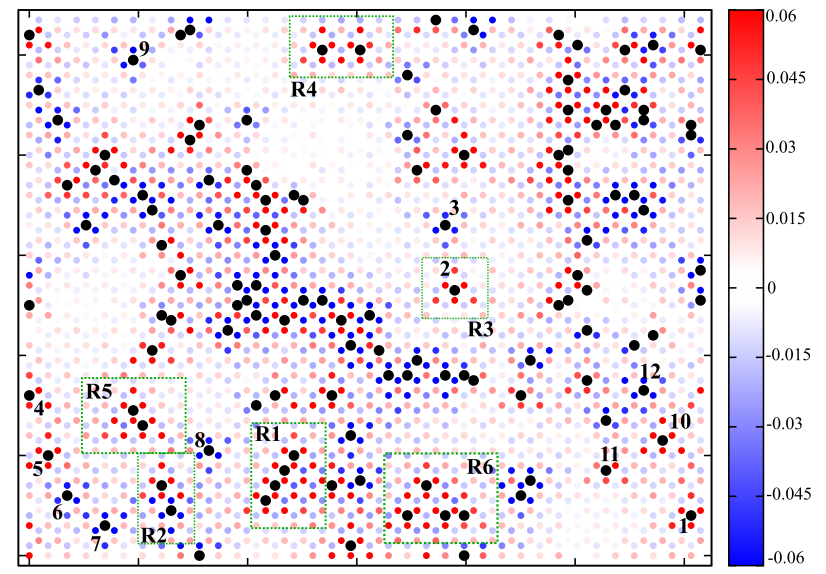

FIG. 2 (color online). Distribution of average spin. The color scale corresponds to $\left\langle S_{z}\right\rangle$ at a site in the zero bare mass limit. 
correlations including explicit evidence for spontaneous breaking of $\mathrm{SU}(2)$ spin symmetry is presented in the Supplemental Material [30].

In order to characterize the correlation between adatoms on one sublattice quantitatively, we have measured the full magnetic moment $M=2 \mu_{B}\left\langle S_{z}\right\rangle$ for some spatial configurations of vacancies. The results (in units of Bohr magnetons) are summarized in the Table I. One can observe a strong dependence of the magnetic moment on the geometry of the adatom configurations. For example, two adatoms at a distance of one lattice step have a magnetic moment 1.5 times larger than two isolated adatoms.

The second set of calculations is devoted to measurements of the mass gap in the presence of vacancies or adatoms. We study two types of energy bands: "normal" energy bands, which transfer into Dirac cones in the absence of adatoms, and midgap states, which are concentrated in the vicinity of isolated adatoms. In the latter case we perform calculations for both sets of potentials to measure the influence of screening on the energies of the midgap states. The calculation of the energies is based on the two-point Euclidean Green's functions contracted with some guess for the projector to the wave function $\psi(x)$ of the state we are interested in

$$
C(\tau)=\sum_{x, y} \operatorname{Tr}\left(\hat{a}_{x}^{\dagger} \bar{\psi}(x) e^{-\tau \hat{H}} \hat{a}_{y} \psi(y) e^{-(\beta-\tau) \hat{H}}\right)
$$

At large enough $\tau$ this correlator is proportional to $e^{-\tau E_{0}}$, where $E_{0}$ is the energy of the state under study. In the case of a normal energy band we use the lattice exponent $\exp (\overrightarrow{i k} \vec{x})$ concentrated at one sublattice with wave vector $\vec{k}$ at the $K$ point of the Brillouin zone as a guess for the wave function. Therefore, we are able to estimate the lower bound of the energy band and the energy gap between these bands. In the case of midgap states, we guess that the wave function is concentrated in the three nearest neighbors of the vacant site. In order to check these measurements we perform the same calculation for freely suspended graphene without vacancies. In this case the gap should be equal to

TABLE I. Average magnetic moment for different configurations of adatoms. R3 corresponds to one isolated adatom, R4 corresponds to two adatoms at a distance of two lattice steps, R5 contains two adatoms at the distance of one lattice step, and R1 and R6 contain four adatoms (denser configuration in the case of R1).

\begin{tabular}{lc}
\hline \hline Region on the map & $M=2\left\langle S_{z}\right\rangle$ \\
\hline R3 & $0.530 \pm 0.016$ \\
R4 & $1.330 \pm 0.026$ \\
R5 & $1.542 \pm 0.026$ \\
R6 & $2.70 \pm 0.04$ \\
R1 & $3.20 \pm 0.04$ \\
\hline \hline
\end{tabular}

zero in the zero bare mass limit [20]. The results for the normal energy band are presented in the inset of Fig. 3. In the presence of vacancies we use a simple linear fit. Without vacancies, the polynomial fit $\phi(m)=c_{0}+c_{1} m+$ $c_{2} m^{2}$ is employed. For the largest lattice $(36 \times 36), c_{0}$ is zero within the error bars, so the fitting works well and this lattice is large enough to reproduce the zero gap in the $m_{\text {bare }} \rightarrow 0$ limit. For smaller lattices one can observe a nonzero $c_{0}$ due to the large finite-size effects.

For the midgap states we used wave functions concentrated near 12 relatively isolated adatoms, marked with black numbers in Fig. 2. Summarizing all of these calculations (see Fig. 3), we conclude that the states concentrated near the adatoms form two rather broad bands between the normal energy bands. The positive and negative energies of the midgap states correspond to adatoms on different sublattices. The gap between these bands is calculated as the distance between the two levels with the smallest absolute values of energies. It can exceed $1 \mathrm{eV}$ for suspended graphene, but decreases for graphene at the substrate. The width of the bands is a measure of the interplay between the midgap states concentrated near different vacancies. Obviously, if the concentration of adatoms tends to zero, the energies of the midgap states will be almost constant. The same effect is observed here in the case of a suppressed Coulomb tail: the midgap states near isolated vacancies recognize their surrounding more poorly.

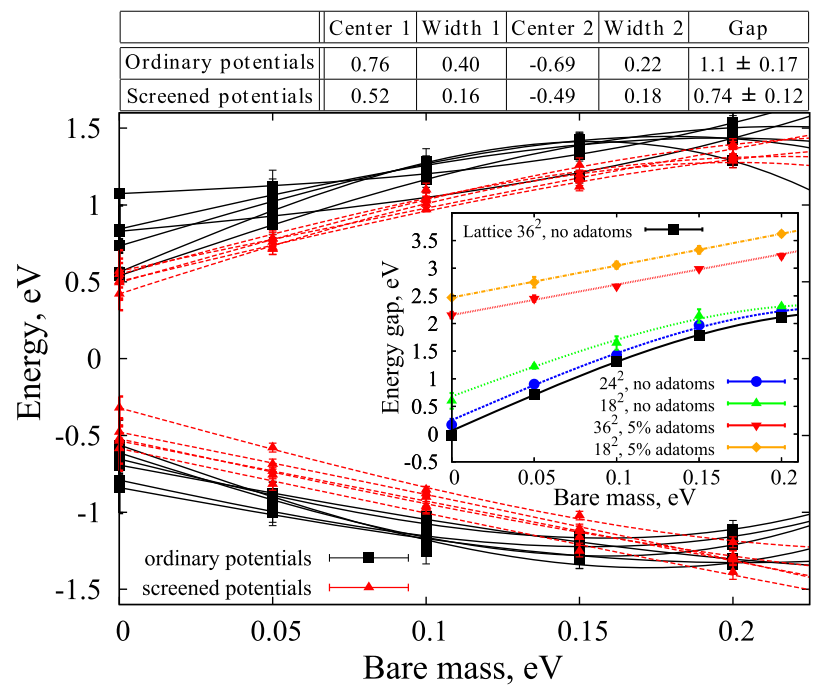

FIG. 3 (color online). Energies of the midgap states for two sets of interelectron potentials. Each state corresponds to one isolated vacancy marked with the number in Fig. 2 . The center and width of the bands are calculated in the limit $m \rightarrow 0$. The center is the average over the energies of all states in each band and the width is equal to the doubled dispersion. $T=0.125 \mathrm{eV}$. The real physical situation is restored in the limit $m \rightarrow 0$. Inset: energy gap between normal energy bands. All values correspond to the $K$ point in the Brillouin zone. 
To conclude, electron-electron interactions for a finite concentration of adatoms lead to antiferromagnetic ordering, in qualitative agreement with the Lieb theorem despite its formal inapplicability to systems with long-range Coulomb interactions. Probably and even more interestingly, they result in a gap opening: a "big gap" of the order of several $\mathrm{eV}$ at the $K$ point and a "smaller gap" (but still quite noticeable, about $1 \mathrm{eV}$ for a $5 \%$ concentration of adatoms) in the midgap states. The latter prediction can be checked by measuring the optics of chemically functionalized graphene. One could expect that the effects of disorder will smear the gap in the defect band transforming it into a pseudogap. One could hope, however, that the twopeak structure characteristic of the pseudogapped state can be distinguished from a single-peak structure centered near the neutrality point predicted by the single-particle picture [29]. Alternatively, the reconstruction of the defect band could be studied experimentally by scanning tunneling spectroscopy.

We thank Dr. P. V. Buividovich for useful discussions. The work of M. V. U. was supported by DFG Grant No. SFB/TR-55 and by Russian Foundation for Basic Research Grant No. 14-02-01261-a. Numerical calculations were performed at the ITEP-FAIR supercomputer center and at the supercomputer center of Moscow State University. M. I. K. acknowledges funding from the European Union Seventh Framework Programme under Grant Agreement No. 604391 Graphene Flagship and from NWO via the Spinoza Prize.

*Maksim.Ulybyshev@physik.uni-regensburg.de ${ }^{\dagger}$ M.Katsnelson@science.ru.nl

[1] N. M. R. Peres, F. Guinea, and A. H. Castro Neto, Phys. Rev. B 73, 125411 (2006).

[2] M. I. Katsnelson, Graphene: Carbon in Two Dimensions (Cambridge University Press, Cambridge, England, 2012).

[3] V. N. Kotov, B. Uchoa, V. M. Pereira, F. Guinea, and A. H. Castro Neto, Rev. Mod. Phys. 84, 1067 (2012).

[4] R. R. Nair, M. Sepioni, I.-L. Tsai, O. Lehtinen, J. Keinonen, A. V. Krasheninnikov, T. Thomson, A. K. Geim, and I. V. Grigorieva, Nat. Phys. 8, 199 (2012).

[5] R. R. Nair, I.-L. Tsai, M. Sepioni, O. Lehtinen, J. Keinonen, A. V. Krasheninnikov, A. H. Castro Neto, M. I. Katsnelson, A. K. Geim, and I. V. Grigorieva, Nat. Commun. 4, 2010 (2013).

[6] P. Haase, S, Fuchs, T. Pruschke, H. Ochoa, and F. Guinea, Phys. Rev. B 83, 241408(R) (2011).
[7] A. K. Mitchell and L. Fritz, Phys. Rev. B 88, 075104 (2013).

[8] T. O. Wehling, S. Yuan, A. I. Lichtenstein, A. K. Geim, and M. I. Katsnelson, Phys. Rev. Lett. 105, 056802 (2010).

[9] O. V. Yazyev and L. Helm, Phys. Rev. B 75, 125408 (2007).

[10] P. M. Ostrovsky, M. Titov, S. Bera, I. V. Gornyi, and A. D. Mirlin, Phys. Rev. Lett. 105, 266803 (2010).

[11] S. Yuan, T. O. Wehling, A. I. Lichtenstein, and M. I. Katsnelson, Phys. Rev. Lett. 109, 156601 (2012).

[12] V. Häfner, J. Schindler, N. Weik, T. Mayer, S. Balakrishnan, R. Narayanan, S. Bera, and F. Evers, Phys. Rev. Lett. 113, 186802 (2014).

[13] T. O. Wehling, E. Şaşıŏglu, C. Friedrich, A. I. Lichtenstein, M. I. Katsnelson, and S. Blügel, Phys. Rev. Lett. 106, 236805 (2011).

[14] W. Han, R. K. Kawakami, M. Gmitra, and J. Fabian, Nat. Nanotechnol. 9, 794 (2014).

[15] T. O. Wehling, E. Şaşığ lu, C. Friedrich, A. I. Lichtenstein, M. I. Katsnelson, and S. Blügel, Phys. Rev. Lett. 106, 236805 (2011).

[16] B. Wang and S. T. Pantelides, Phys. Rev. B 86, 165438 (2012).

[17] A. V. Shytov, D. A. Abanin, and L. S. Levitov, Phys. Rev. Lett. 103, 016806 (2009).

[18] F. Gargiulo, G. Autès, N. Virk, S. Barthel, M. Rösner, L. R. M. Toller, T. O. Wehling, and O. V. Yazyev, Phys. Rev. Lett. 113, 246601 (2014).

[19] P. V. Buividovich and M. I. Polikarpov, Phys. Rev. B 86, 245117 (2012).

[20] M. V. Ulybyshev, P. V. Buividovich, M. I. Katsnelson, and M. I. Polikarpov, Phys. Rev. Lett. 111, 056801 (2013).

[21] J. E. Drut and T. A. Lahde, Phys. Rev. Lett. 102, 026802 (2009).

[22] W. Armour, S. Hands, and C. Strouthos, Phys. Rev. B 81, 125105 (2010).

[23] R. C. Brower, C. Rebbi, and D. Schaich, arXiv:1101.5131; Proc. Sci., LAT2011 (2012) 056 [arXiv:1204.5424].

[24] D. Smith and L. von Smekal, Phys. Rev. B 89, 195429 (2014).

[25] H. De Raedt and A. Lagendijk, Phys. Rep. 127, 233 (1985).

[26] E. H. Lieb, Phys. Rev. Lett. 62, 1201 (1989).

[27] S. Just, S. Zimmermann, V. Kataev, B. Büchner, M. Pratzer, and M. Morgenstern, Phys. Rev. B 90, 125449 (2014).

[28] A. N. Rudenko, F. J. Keil, M. I. Katsnelson, and A. I. Lichtenstein, Phys. Rev. B 88, 081405(R) (2013).

[29] S. Yuan, H. De Raedt, and M. I. Katsnelson, Phys. Rev. B 82, 115448 (2010).

[30] See Supplemental Material athttp://link.aps.org/ supplemental/10.1103/PhysRevLett.114.246801 for explicit evidence of spontaneous breaking of SU(2) spin symmetry and for the description of spin-spin correlations for various geometrical configurations of adatoms. 